\title{
Flipped Classroom y el aprendizaje de condicionales en inglés en estudiantes Universitarios de nivel intermedio.
}

\section{Flipped Classroom and conditionals learning in English to University students of intermedia level}

Lina Yolanda Morales Rodas, ${ }^{1} \&$, Jessica Valentina Galimberti. ${ }^{2}$

\section{Recibido: 25-02-2019 / Revisado: 28-02-2019 /Aceptado: 24-03-2019/ Publicado: 05-06-2019}

\begin{abstract}
.
DOI: $\quad$ https://doi.org/10.33262/cienciadigital.v3i3.652

The research, "Flipped Classroom and the Learning of Conditionals in English Intermediate-level University Students," carried out at Escuela Superior Politécnica de Chimborazo, campus Macas, aimed to analyze the incidence of the application of the Inverted Classroom technique in English writing production when structuring conditional sentences. The population of this quasi-experimental study was formed by sixteen students in the experimental group and sixteen students in the control group. In the first phase of the research, a pre-test was applied to both groups, then the Flipped Classroom technique was implemented for four weeks, in the English classes of the experimental group. Finally, a post-test on conditionals was given to both groups from which errors were collected to verify the proposition of the research. The data obtained was tabulated and analyzed, determining the highest incidence of linguistic errors and the effectiveness of using the inverted classroom as a teaching-learning technique.
\end{abstract}

Keywords: Flipped Classroom, Conditional Sentences, Linguistic Errors

\section{Resumen.}

La presente investigación, "Flipped Classroom y el aprendizaje de condicionales en Inglés en estudiantes Universitarios de nivel intermedio", se la realizó en la Escuela Superior Politécnica de Chimborazo Sede Macas, con el propósito de analizar la incidencia que tiene la aplicación de la técnica de Aula Invertida en la producción escrita en Inglés, específicamente al estructurar oraciones condicionales. Esta es una

1 Escuela Superior Politécnica de Chimborazo, Sede Morona Santiago. Macas, Ecuador lina.morale@espoch.edu.ec

2 Escuela Superior Politécnica de Chimborazo, Sede Morona Santiago. Macas, Ecuador. jessica.galimberti@espoch.edu.ec 
investigación cuasi-experimental, en la que la población está conformada por dos grupos de dieciséis estudiantes en cada uno, un experimental y otro de control. En la primera fase de la investigación se aplicó una prueba de diagnóstico denominada pretest a ambos grupos de inglés de nivel intermedio, para luego implementar la técnica Flipped Classroom durante cuatro semanas únicamente con el grupo experimental para la estructuración de oraciones condicionales, mientras que el grupo de control recibió clases tradicionales sobre el mismo tema. Para verificar la propuesta de la investigación se aplicó un post-test sobre condicionales a ambos grupos de donde se recopilaron datos sobre los errores observados. La información obtenida fue tabulada y analizada determinado cuáles fueron los errores lingüísticos con mayor incidencia y la efectividad de utilizar el aula invertida como técnica de enseñanza-aprendizaje.

Palabras claves: Aula Invertida, Oraciones Condicionales, Errores Lingüísticos

\section{Introducción.}

La tecnología avanza y mejora cada día, de igual forma la manera de aprender de las nuevas generaciones cambia de acuerdo a la actualización de dicha tecnología. El proceso de educar es un ente activo en constante renovación, pero Juhary, \& Amir (2018) acota que, bajar información de la WEB para utilizarla en el aula de clase no es significado de una educación tecnificada, los educadores deben estar en constante aprendizaje, más aún sobre cómo utilizar apropiadamente la tecnología

El uso del aula invertida como alternativa a los entornos de aprendizaje tradicionales ha sido de mucho interés ya que cada vez atrae más la atención de investigadores y educadores. El avance en la tecnología, herramientas tales como videos interactivos, actividades interactivas en clase y sistemas de videoconferencia allanan el camino para el uso generalizado de flipped classroom. Estudios sobre el flipped classroom aparecen en diferentes disciplinas, incluidos los sistemas de información Davies, Dean \& Ball (2013)., ingeniería, sociología y humanidades Kim, Kim, Khera, \& Getman (2014), matemáticas educación Zengin (2017)., y composición en inglés.

Básicamente lo que se distingue de las clases tradicionales es el hecho de poner en primer lugar el estudio del argumento en la casa por parte de los estudiantes, usando lecciones en video preparadas por el profesor o terceros. Como consecuencia, en clase los alumnos aplican el conocimiento resolviendo problemas y haciendo trabajos prácticos. Las ventajas del método tradicional y del nuevo se combinan, el aspecto principal es saber y poder optimizar el tiempo. El docente enseña a los alumnos a través de la práctica y repaso o explicación más profunda de los conceptos ya vistos por su cuenta. Los alumnos aprenden haciendo y se ayudan mutuamente así que se convierte también en un proceso que beneficia tanto al avanzado y también al estudiante menos avanzado. La ayuda que el profesor da con respecto al método tradicional es la de gastar su tiempo trabajando con estudiantes que los necesitan y juntos resolver los problemas.

Dentro de las ventajas del aula invertida que Zainuddin \& Halili (2016) menciona en su trabajo "Flipped Classroom Research and Trends from Different Fields of Study" está que aprender a través de una clase invertida hace que los estudiantes aprendan mejor debido a 
que este proceso lo pueden desarrollar a su propio ritmo y estimula en el estudiante su necesidad de seguir auto-educándose.

Santikarn \& Wichadee (2018). en su investigación "Flipping the Classroom for English Language Learners: A Study of Learning Performance and Perceptions" asevera que, según la percepción de los estudiantes el nuevo ambiente de aprendizaje que experimentaron con la utilización del aula invertida fue muy satisfactoria y encontraron muchas ventajas como la motivación que encontraron en darse cuenta que podían realizar con mayor precisión tareas online y tareas grupales en clase sobre el tema tratado.

El propósito de este estudio de investigación es analizar la eficacia en voltear el contenido relacionado a los condicionales. Bennett \& Bennett (2003) expresa que, los condicionales son oraciones que hablan de un posible escenario en el que se da, se dará o pudo haberse dado un resultado. Una oración condicional está formada de dos partes: un antecedente, premisa o condición y una consecuencia o resultado. Existen cuatro tipos de condicionales que se distinguen claramente por el hecho de que el resultado sea una situación que se pueda realizar o que sea una situación imposible, desde el cero condicional cuya condición está en un tiempo presente y el resultado es real y se da en todos los casos posibles, hasta el tercer condicional en el cual la condición está en un pasado perfecto y el resultado es imposible.

Estructurar oraciones condicionales en inglés forma parte de la producción escrita, en la que los estudiantes al aprender una segunda lengua o una lengua extranjera cometen errores, mismos que han sido motivo de interés y estudio por varios expertos en la materia, entre ellos Corder, quien propuso "describir, clasificar, y explicar los errores producidos por los aprendices de una L2 comparando su actuación intralingüística con la lengua meta”. Este autor identifica cuatro tipos de error linguiístico: la adición, la omisión, la selección falsa o elección incorrecta, y la colocación falsa. Por otro lado, Jame (1998), hace la misma clasificación de los errores lingüísticos con la siguiente variación: errores de adición, errores de omisión, errores por forma errónea, errores de mal ordenamiento y errores de mezcla

\section{Metodología}

Con el fin de lograr el objetivo de la investigación que fue la de determinar la incidencia de aplicar la técnica de enseñanza Flipped Classroon (Aula Invertida) en la comprensión de condicionales en Inglés, se empleó un enfoque cualitativo, cuantitativo.

En forma cualitativa se realizó una recolección y análisis de información estrechamente relacionada a la parte teórica del problema, lo cual permitió entender y poder aplicar la técnica del aula invertida (Flipped Classroom) para la enseñanza del Idioma Inglés, específicamente los condicionales en la producción escrita.

El enfoque cuantitativo permitió determinar la incidencia que tuvo la aplicación del aula invertida durante la fase de intervención, en los estudiantes de nivel intermedio de la ESPOCH Sede Morona Santiago sobre la comprensión de los condicionales en inglés, luego que se realizó la aplicación del pre-test y post test, con los que se pudo determinar los resultados finales. 
La presente investigación fue de tipo bibliográfico y de campo. Se recopiló información tanto de literatura impresa y digital, mismas que se referenciaron en este estudio. Por otro lado, los datos que se obtuvieron en este estudio fueron recabados directamente de los test aplicados a los estudiantes, antes (pre-test) y después (post-test) de la fase de intervención (enseñanza aplicando la técnica del Flipped-Classroom)

Gráfico 1. Fases de la Investigación
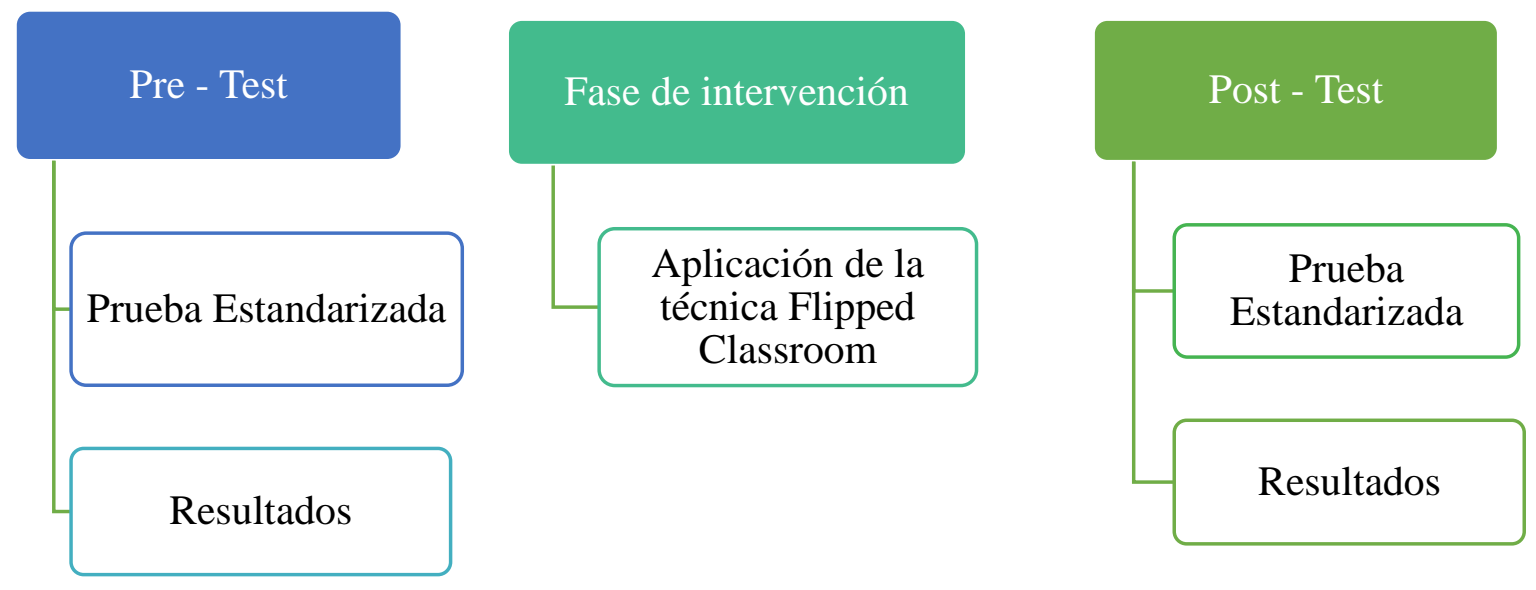

Fuente: Trabajo bibliográfico

Elaborado por: Las autoras

El nivel de profundidad de este estudio es de tipo correlacional, se tiene una variable independiente que es la aplicación de la técnica de aula invertida (Flipped Classroom), misma que tiene incidencia en la variable dependiente que es la comprensión de condicionales en inglés en los estudiantes de nivel intermedio (B1) de la ESPOCH Sede Morona Santiago.

La investigación realizada en la ESPOCH Sede Morona Santiago, es de carácter cuasiexperimental debido a que la población conformada por 32 casos, no fue seleccionada al azar. Se consideraron dos grupos de estudiantes de inglés de nivel intermedio (B1) de acuerdo al Marco Común Europeo de Referencia (CEFR), uno de control y otro experimental, en ambos casos trabajaron directamente las investigadoras.

Tabla 1. Población

\begin{tabular}{lccc}
\hline \multicolumn{1}{c}{ Informantes } & Horario & Frecuencia & Porcentaje \\
\hline $\begin{array}{l}\text { Grupo de Control de Nivel } \\
\text { Intermedio - Minas (B1) }\end{array}$ & $09: 00-11: 00$ & 16 & $100 \%$ \\
$\begin{array}{l}\text { Grupo Experimental de Nivel } \\
\text { Intermedio - Ambiental (B1) }\end{array}$ & $11: 00-13: 00$ & 16 & $100 \%$ \\
\hline
\end{tabular}

Fuente: Área de Inglés ESPOCH Sede Morona Santiago

Elaborado por: Las autoras 
En la primera fase de esta investigación se aplicó a ambos grupos un test de diagnóstico (pretest), con el objetivo de determinar el conocimiento previo que tenían los estudiantes sobre los condicionales. La segunda fase fue la de aplicación de la técnica Flipped Classroom en el proceso de enseñanza-aprendizaje de condicionales, al grupo experimental, luego de la cual se aplicó un segundo test (post-test) a ambos grupos para obtener los resultados luego del período de intervención.

Este estudio propone la aplicación de la técnica de aula invertida para la producción escrita de condicionales en inglés durante un tiempo determinado de cuatro semanas, dando así el carácter longitudinal a la investigación.

Se empleó el baremo o regla para medir e interpretar puntuaciones para determinar las frecuencias de los diferentes errores lingüísticos que se produjeron en las pruebas escritas de los estudiantes. Luego se realizó un análisis descriptivo y estadístico de los datos obtenidos, para determinar la frecuencia de repetición de los errores linguísticos, porcentajes y media aritmética en el pre-test y en el post-test de los estudiantes, tanto del grupo de control como del grupo experimental.

Los errores lingüísticos de la producción escrita analizados y clasificados en el presente trabajo son errores de adición, de omisión, por forma errónea, de mal ordenamiento, y de mezcla.

Tabla 2. Errores lingüísticos analizados en la producción escrita en inglés.

Criterio Descripción

Errores de adición

Regularización: pasar por alto excepciones que tienen ciertas reglas gramaticales

Doble marca: no eliminar ciertos elementos innecesarios en ciertas estructuras gramaticales

Adición Simple: cualquier tipo de adición diferente a la de regularización o doble marca

Errores de omisión

Errores por forma errónea

Errores de mal ordenamiento

Errores de mezcla
Ausencia de un morfema o de una palabra

Utilización en forma errónea de una estructura en lugar de otra que es correcta

Colocación en forma incorrecta de un morfema, un conjunto de morfemas o elementos de la oración modificando el orden sintagmático

Cuando se tienen dos estructuras que podrían servir e inconscientemente se hace una mezcla de las dos

Fuente: Trabajo bibliográfico

Elaborado por: Las autoras 


\section{Resultados}

Luego de que se aplicaron el pre-test y el post-test, se tabularon los datos tanto de los errores analizados en los test como de las notas que obtuvieron tanto los estudiantes del grupo de control como los del grupo experimental.

Tabla 3. Errores determinados en el Pre-Test

\begin{tabular}{lcccc}
\hline & \multicolumn{2}{c}{ Grupo de Control } & \multicolumn{2}{c}{ Grupo Experimental } \\
\hline Criterio & Frecuencia & Porcentaje & Frecuencia & Porcentaje \\
$\begin{array}{l}\text { Errores de } \\
\text { adición }\end{array}$ & 6 & $7.1 \%$ & 0 & $0 \%$ \\
$\begin{array}{l}\text { Errores de } \\
\text { omisión }\end{array}$ & 13 & $15.3 \%$ & 13 & $13.4 \%$ \\
$\begin{array}{l}\text { Errores por forma } \\
\text { errónea }\end{array}$ & 27 & $31.8 \%$ & 69 & $71.1 \%$ \\
$\begin{array}{l}\text { Errores de mal } \\
\text { ordenamiento }\end{array}$ & 33 & $38.8 \%$ & 12 & $12.4 \%$ \\
Errores de mezcla & 6 & $7 \%$ & 3 & $3.1 \%$ \\
TOTAL & & & & \\
Media X & $\mathbf{8 5}$ & $\mathbf{1 0 0 \%}$ & $\mathbf{9 7}$ & $\mathbf{1 0 0 \%}$ \\
\hline
\end{tabular}

En la tabla 3 se ha tabulado la información analizada del pre-test aplicado tanto al grupo de control como al grupo experimental, según estos datos los errores lingüísticos que más incidencia tuvieron fueron los de forma errónea. Dentro de estos errores se pudo detectar que los estudiantes el estructurar correctamente los componentes de los condicionales: cláusula de condición y cláusula de resultado, en los tiempos de conjugación correspondiente a cada uno. 
Gráfico 2. Errores lingüísticos analizados pre-test Grupo de Control y Experimental

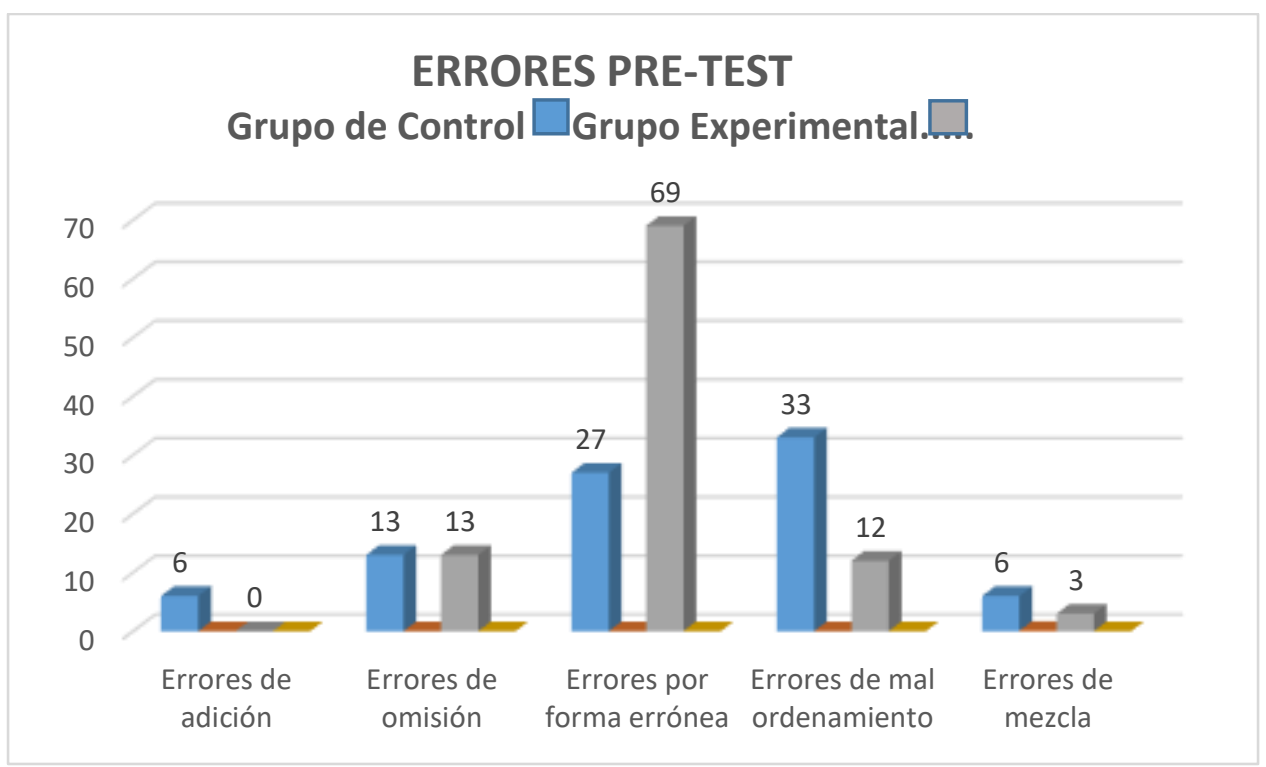

Gráfico 3. Cantidad de errores analizados en el pre-test Grupo de Control y Experimental

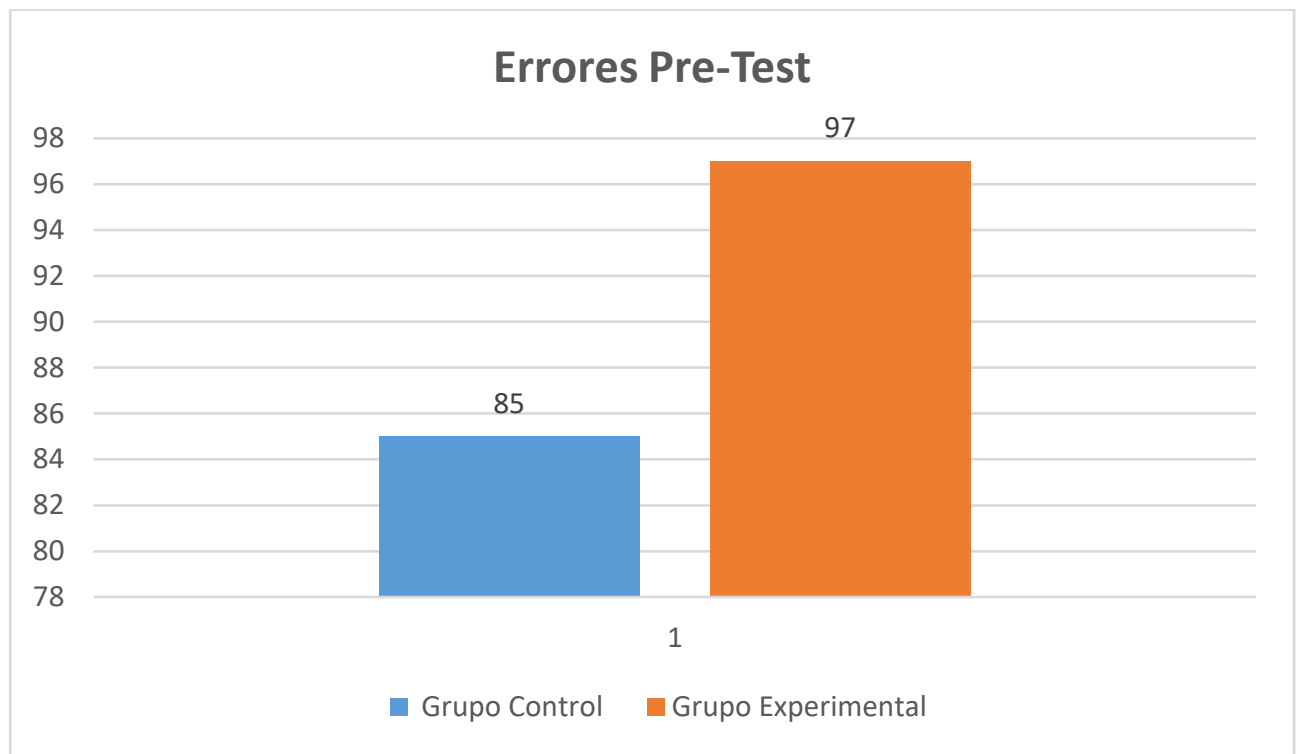

Los datos que se muestran en el gráfico 3 indican que en el test diagnóstico aplicado tanto al grupo de control como al grupo experimental se han cometido errores lingüísticos, indicando el gráfico que en el grupo experimental se detectaron más errores que en el grupo de control. 
Tabla 4. Errores determinados en el Post-Test

\begin{tabular}{lcccc}
\hline & \multicolumn{2}{c}{ Grupo de Control } & \multicolumn{2}{c}{ Grupo Experimental } \\
\hline Criterio & Frecuencia & Porcentaje & Frecuencia & Porcentaje \\
$\begin{array}{l}\text { Errores de } \\
\text { adición }\end{array}$ & 1 & 1.5 & 0 & $0 \%$ \\
$\begin{array}{l}\text { Errores de } \\
\text { omisión }\end{array}$ & 4 & 5.8 & 8 & $17 \%$ \\
$\begin{array}{l}\text { Errores por forma } \\
\text { errónea }\end{array}$ & 53 & 76.8 & 24 & $51.1 \%$ \\
$\begin{array}{l}\text { Errores de mal } \\
\text { ordenamiento }\end{array}$ & 18 & 14.5 & 15 & $31.9 \%$ \\
Errores de mezcla & 1 & 1.5 & & \\
TOTAL & & $\mathbf{1 0 0 \%}$ & $\mathbf{4 7}$ & $\mathbf{1 0 0 \%}$ \\
Media X & $\mathbf{7 7}$ & & $\mathbf{2 . 9}$ & \\
\hline
\end{tabular}

Gráfico 4. Errores lingüísticos analizados pre-test Grupo de Control y Experimental

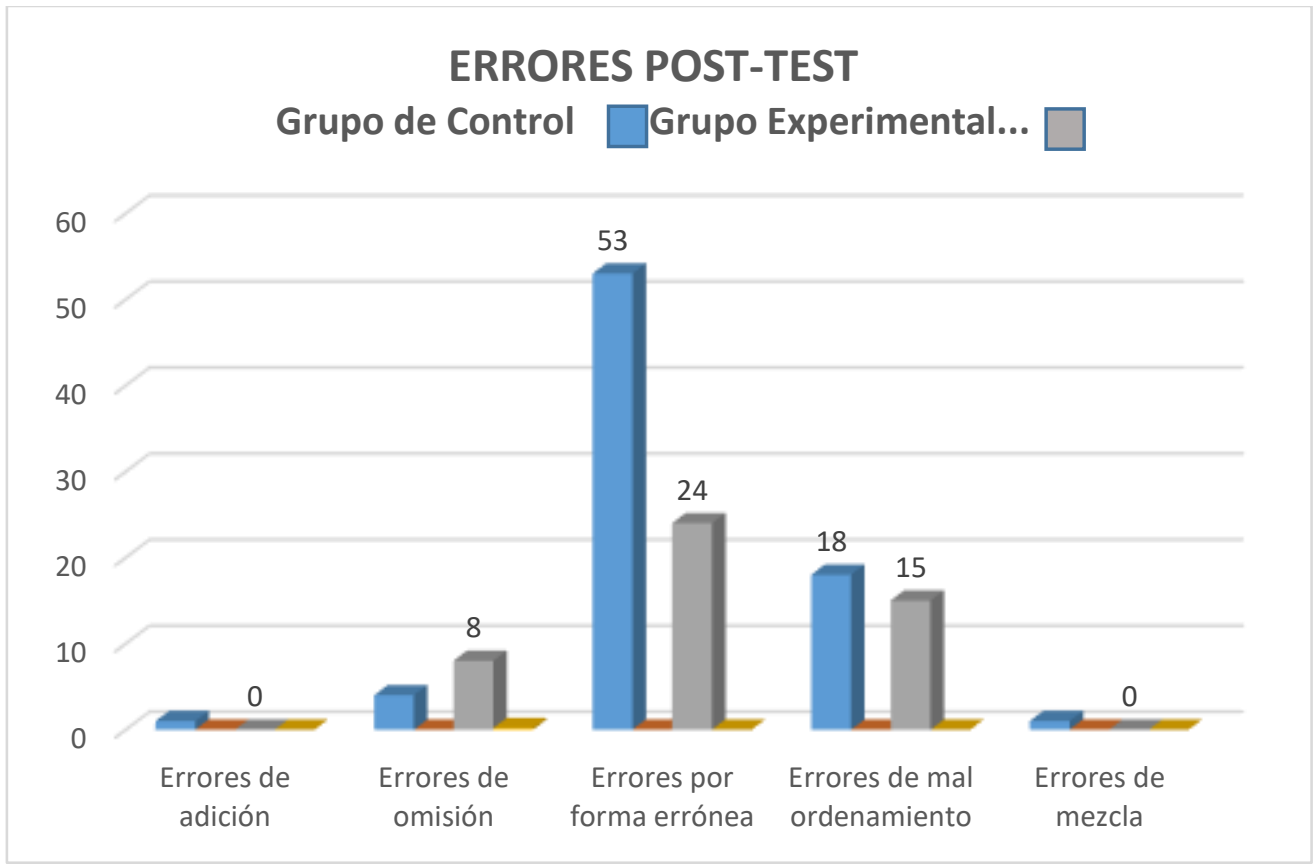


Gráfico 5. Cantidad de errores analizados en el post-test Grupo de Control y Experimental

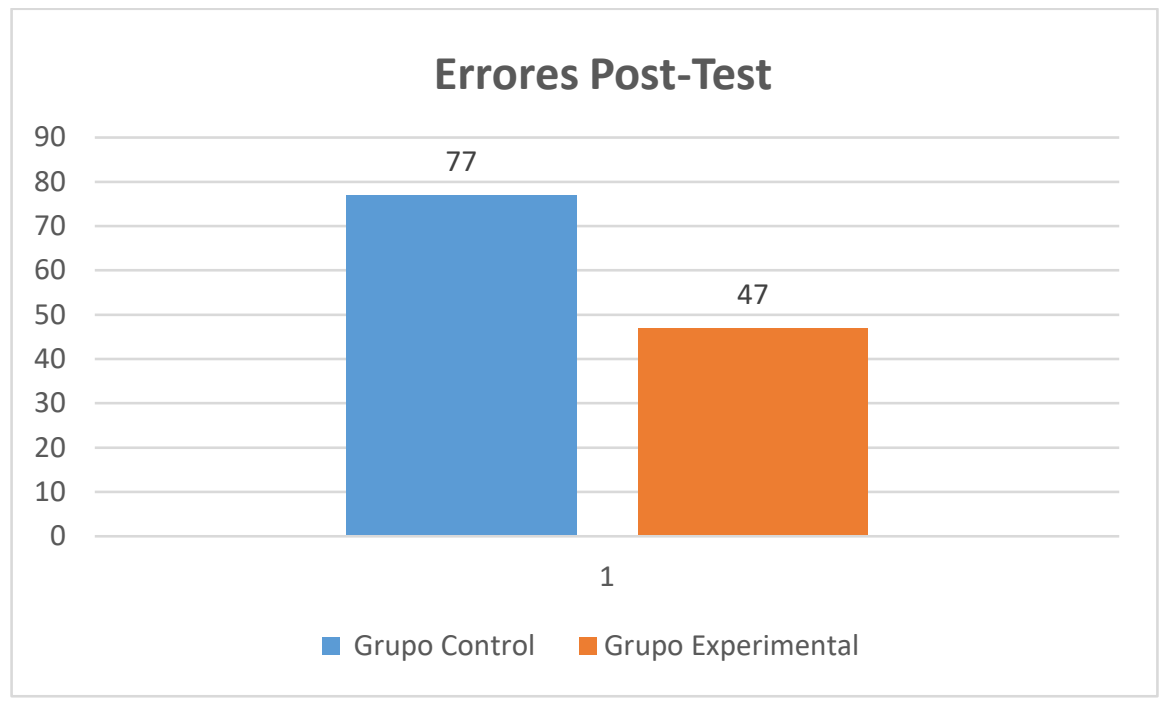

En los gráficos 4 y 5 se muestran los resultados obtenidos luego de la aplicación del post test tanto al grupo experimental como de control. Con los resultados se observa que luego de la aplicación de la técnica de aula invertida (Flipped Classroom), el grupo experimental cometió menos errores que el grupo de control, por lo tanto, esto significa que la técnica de enseñanza Flipped Classroom tuvo un impacto favorable en la producción escrita de condicionales en Inglés y puede ser considerada como una técnica que promueve un aprendizaje significativo.

\section{Discusión}

Mediante la investigación se constató que durante el proceso enseñanza-aprendizaje de una segunda lengua se cometen errores lingüísticos en la producción escrita, siendo según las tablas de resultados, los errores por forma errónea los más comunes, esto debido a la interferencia del español como lengua materna en la estructuración de oraciones gramaticales en la segunda lengua en este caso el Inglés.

Se pudo establecer mediante el test diagnóstico (pre-test) que los estudiantes universitarios cuentan con conocimientos previos, adquiridos en niveles anteriores. Los datos de la tabla 3 nos demuestran que el grupo experimental cometió un número mayor de errores que el grupo de control.

A pesar de emplear técnicas efectivas para el aprendizaje como el aula invertida, se siguen percibiendo interferencias gramaticales de la lengua materna en la segunda lengua, mismas 
que se reflejan en los errores cometidos por el grupo experimental en la estructuración de oraciones condicionales en el post-test.

El aula invertida es una técnica que hace que el estudiante comprenda conceptos por si mismo de una manera más dinámica y con la participación total del mismo.

\section{Conclusiones.}

- Se ha establecido la correlación entre la aplicación del aula invertida y la producción escrita en Inglés, concluyendo que al aplicar el aula invertida como técnica de enseñanza se obtuvieron menos errores en la producción escrita de oraciones condicionales.

- En relación al tipo de errores lingüísticos que los estudiantes cometen, se determinó con la aplicación del pre-test que, los de mayor incidencia son los errores por forma errónea, mismos que se dan por la utilización de una estructura en lugar de otra que es correcta

- Con una frecuencia menor se pudieron observar errores de mal ordenamiento, de omisión, de mezcla y de adición.

- Al realizar el análisis de resultados obtenidos luego de la aplicación del post-test se determinó que mientras el grupo experimental muestra resultados positivos con la aplicación del aula invertida, el grupo de control a pesar de los esfuerzos realizados por el docente quien aplicó procesos tradicionales de enseñanza, no pudo disminuir en la misma cantidad los errores cometidos en la producción escrita en inglés

- De acuerdo a los datos obtenidos, se puede concluir que la utilización de la técnica del aula invertida es eficaz para que se dé un aprendizaje significativo de las oraciones condicionales

- El aula invertida representa una alternativa de enseñanza del idioma Inglés, mediante la cual los estudiantes adquieren conocimientos más sólidos reflejándose en la disminución de errores al estructurar oraciones condicionales.

\section{Referencias bibliográficas.}

- Bennett, J. F., \& Bennett, J. (2003). A philosophical guide to conditionals. Oxford University Press on Demand.

- Corder, S. P. (1982). Error analysis and interlanguage (Vol. 198, No. 1). Oxford University Press.Davies, R. S., Dean, D. L., \& Ball, N. (2013). Flipping the classroom and instructional technology integration in a college-level information systems spreadsheet course. Educational Technology Research and Development, 61(4), 563580.James, C. (2013). Errors in language learning and use: Exploring error analysis.

- Routledge.Juhary, J., \& Amir, A. F. (2018, July). Flipped Classroom at the Defence University. In 4th International Conference on Higher Education Advances (HEAd'18) (pp. 827-835). Editorial Universitat Politècnica de València. 
- Kim, M. K., Kim, S. M., Khera, O., \& Getman, J. (2014). The experience of three flipped classrooms in an urban university: an exploration of design principles. The Internet and Higher Education, 22, 37-50.

- Santikarn, B., \& Wichadee, S. (2018). Flipping the Classroom for English Language Learners: A Study of Learning Performance and Perceptions. International Journal of Emerging Technologies in Learning (iJET), 13(09), 123-135.

- Zengin, Y. (2017). Investigating the use of the Khan Academy and mathematics software with a flipped classroom approach in mathematics teaching. Journal of Educational Technology \& Society, 20(2), 89-100.

- Zainuddin, Z., \& Halili, S. H. (2016). Flipped classroom research and trends from different fields of study. The International Review of Research in Open and Distributed Learning, 17(3). 


\section{PARA CITAR EL ARTÍCULO INDEXADO.}

Morales Rodas, L. Y., \& Galimberti, J. (2019). Flipped Classroom y el aprendizaje de condicionales en inglés en estudiantes Universitarios de nivel intermedio. Ciencia Digital, 3(3), 386-397. https://doi.org/10.33262/cienciadigital.v3i3.652

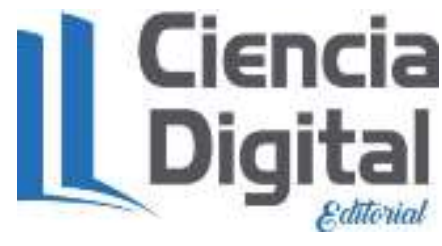

El artículo que se publica es de exclusiva responsabilidad de los autores y no necesariamente reflejan el pensamiento de la Revista Ciencia Digital.

El artículo queda en propiedad de la revista y, por tanto, su publicación parcial y/o total en otro medio tiene que ser autorizado por el director de la Revista Ciencia Digital.
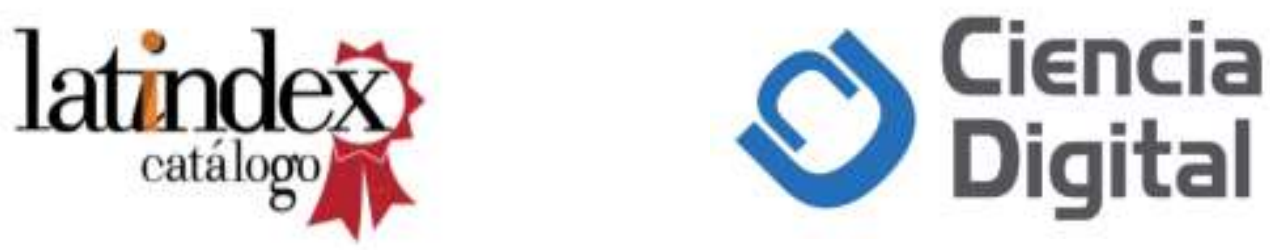